\title{
Efeito da Conexidade de Dados sobre a Acurácia dos Testes de Progênie e Performance ${ }^{1}$
}

\author{
Antonio Policarpo Souza Carneiro 2,5 , Robledo de Almeida Torres ${ }^{3,6}$, Ricardo Frederico \\ Euclydes $^{3}$, Martinho de Almeida e Silva 4, 6 , Paulo Sávio Lopes ${ }^{3,6}$, Paulo Luiz Souza Carneiro ${ }^{2,6}$, \\ Rodolpho de Almeida Torres Filho2, 6
}

\begin{abstract}
RESUMO - Dados simulados foram utilizados para verificar o efeito da conexidade sobre a acurácia dos testes de progênie e performance. O genoma simulado foi constituído de uma característica quantitativa governada por 500 locos. Simulou-se o efeito de rebanho (9 rebanhos) significativo a 5\% de probabilidade pelo teste F. Foram simulados arranjos de dados com 0, 15, 30, 60, 90 e 100\% de conexidade para herdabilidades 0,$10 ; 0,30$; e 0,60 e para diferentes tamanhos de progênie ( 9,54 e 90 progênies/reprodutor). A cada geração foram selecionados nove machos e o número de fêmeas selecionadas variou de acordo com o número de progênies considerado em cada arranjo de dados. A seleção foi efetuada em dez gerações, sendo este processo repetido por 30 vezes. O uso de dados com baixa conexidade reduziu a acurácia dos testes considerados, e a maior importância da conexidade ocorreu para baixa herdabilidade e reduzido tamanho da progênie. Apesar de ter sido observado efeito da conexidade, a herdabilidade e o tamanho da progênie tiveram maior influência na avaliação genética.
\end{abstract}

Palavras-chave: avaliação genética, BLUP, seleção e simulação

\section{Data Connectedness Effect on the Accuracy of Progeny and Performance Tests}

\begin{abstract}
Simulated data were used to verify the effect of the connectedness on the accuracy of progeny and performance tests. The simulated genome was formed from a quantitative trait governed by 500 loci. Significance herd effect ( 9 herds) by $5 \%$ probability F test was simulated. Data sets with $0,15,30,60,90$ and $100 \%$ of connectedness for heritabilities $0.10,0.30$ and 0.60 and for different progeny sizes ( 9,54 and 90 progenies/sire) were simulated. In each generation nine males were selected and the number of selected females shifted according to the number of progenies considering in each data arrange. The selection was accomplished in ten generations, where the process was repeated 30 times. Data with smaller connectedness reduced the accuracy of both tests and the connectedness was more important for small heritability and small progeny size. The heritability and the progeny size had larger influence in the genetic evaluation than the effect of connectedness.
\end{abstract}

Key Words: BLUP, genetic evaluation, selection e simulation

\section{Introdução}

No Brasil, tem crescido a conscientização em relação à importância da utilização do teste de progênie para avaliação genética de animais, representando uma mudança em direção à eficiência na seleção. A parceria entre criadores de animais de elite e instituições de ensino e pesquisa tem promovido maior engajamento dos criadores em programas de avaliações genéticas.

A conexidade genética entre dois rebanhos é estabelecida pelo parentesco entre os animais pertencentes a estes rebanhos. O uso da inseminação artificial contribui para aumentar a precisão do teste de progênie, proporcionando progênies mais numerosas e permitindo melhor distribuição das progênies entre rebanhos, aumentando a conexidade dos dados.

$\mathrm{Na}$ avaliação genética em diferentes rebanhos, a conexidade dos dados é um dos fatores que afeta a acurácia do teste de progênie. A baixa conexidade, devido aos reprodutores terem filhos em um número pequeno de rebanhos, por exemplo, contribui para aumentar as diferenças sistemáticas entre os grupos de progênies, comprometendo a acurácia da avaliação genética.

A conexidade entre os dados, juntamente com a

\footnotetext{
${ }^{1}$ Parte da tese de Mestrado do primeiro autor. Financiamento: CAPES/CNPq/UFV.

${ }^{2}$ Estudante de Pós-graduação da UFV. 36571-000 - Viçosa-MG. E.mail: policarpoc@bol.com.br

3 Prof. da UFV. Depto. de Zootecnia - CCA, Viçosa-MG. E.mail: rtorres@mail.ufv.br; rbaja@mail.ufv.br; pslopes@mail.ufv.br

4 Professor da UFMG, Belo Horizonte-MG. E.mail: martinho@vet.ufmg.br

5 Bolsista da CAPES. E.mail: ds31954@correio.cpd.ufv.br

6 Bolsista do CNPq. E.mail: ds28686@correio.cpd.ufv.br
} 
ocorrência de seleção e da interação genótipo-ambiente, é uma das limitações ao uso das Equações de Modelos Mistos de Henderson (EMMH) para a obtenção do BLUP dos valores genéticos dos animais (MARTINS, 1994).

SCHENKEL (1991), analisando as vantagens do uso da metodologia de modelos mistos, citou que o problema de conexidade não é identificado nesta metodologia. O processo faz com que as soluções dentro dos subgrupos desconectados somem a zero e, portanto, não provocam colapso no sistema, pressupondo que não existam diferenças genéticas entre os subgrupos, o que nem sempre é verdade. Esta pode ser considerada uma desvantagem do método, uma vez que, se os subgrupos somam zero, não poderia haver comparações entre animais de diferentes subgrupos desconectados.

FRIES e ROSO (1997) criticaram a aplicação das EMMH que sempre fornecem soluções, para qualquer estrutura de dados, não existindo qualquer exigência mínima de conexidade, nem um padrão mínimo de referência, e nem uma forma universal de medir o grau de conexidade dos dados.

Os países importadores de sêmen deparam-se com um grande problema na escolha de touros para utilizar nos seus rebanhos, uma vez que cada país exportador faz sua própria avaliação genética para diferentes características, metodologias e normas. Vários autores relataram a importância das avaliações genéticas internacionais, visando contornar estes problemas (PHILIPSSON, 1987; Banos e Smith, 1991; Goddard, 1992 e Burnside et al., 1992, citados porBANOS, 1994).

A conexidade de dados na avaliação genética faz parte de um problema mais geral de ajustamento para efeito de rebanho e rebanho-ano-estação, e de estimação de médias genéticas das populações, ou seja, de estimação dos efeitos de grupo genético (FOULLEY et al., 1987). KENNEDY e TRUS (1993) e BANOS (1994) relataram a influência da conexidade dos dados sobre a precisão das avaliações genéticas globalizadas.

Este trabalho teve como objetivo avaliar o efeito da conexidade de dados sobre a acurácia do teste de progênie e do teste de performance, para diferentes números de progênie por reprodutor e para características com diferentes herdabilidades.

\section{Material e Métodos}

Para a simulação dos dados, foi utilizado o programa "Genesys"(Genetic System) desenvolvidoporEUCLYDES (1996), escrito para o compilador FORTRAN.
Foi simulado um genoma constituído de 15 pares de cromossomos. As características quantitativas usadas neste estudo foram controladas por 500 locos, distribuídos ao acaso nos cromossomos. Os locos quantitativos foram dialélicos e não possuíam desvios de dominância e nem interações entre si. Foi simulado o efeito aditivo de cada gene, tendo estes efeitos uma distribuição normal.

Cada uma das três populações-base simuladas foi constituída por 1800 animais, sendo 900 machos e 900 fêmeas. A cada população-base foi associada uma característica de herdabilidade definida, sendo simuladas características com herdabilidades baixa, média e alta $(0,10 ; 0,30 ;$ e 0,60 , respectivamente) e, além do efeito fixo de sexo, foi simulado o efeito de rebanho com nove níveis. Os efeitos de rebanho foram simulados, de modo a produzir significância a $5 \%$ de probabilidade pelo teste $\mathrm{F}$.

A partir de cada população-base, foram simuladas populações com diferentes números de progênies/ reprodutor: 9, 54 e 90 (Tabela 1). Nove machos e 27, 162 ou 270 fêmeas foram escolhidos aleatoriamente em cada população-base para serem os pais das três populações iniciais.

A avaliação genética dos animais iniciou-se a partir das populações iniciais, onde foram selecionados nove machos e um número determinado de fêmeas, de acordo com o número de progênies/reprodutor (Tabela 1). A seleção foi praticada por dez gerações e foram feitas 30 repetições para reduzir o efeito da flutuação gênica.

Foram simulados arranjos de dados com 0, 15, 30, 60,90 e $100 \%$ de conexidade média. Estes arranjos foram obtidos variando o número de rebanhos em que cada reprodutor tinha progênies. Para $0 \%$ de conexidade, os reprodutores tinham progênies em um único rebanho e para $100 \%$ de conexidade, as progênies de cada reprodutor estavam distribuídas nos nove rebanhos simulados. Nos arranjos com grau intermediário de conexidade, as progênies estavam

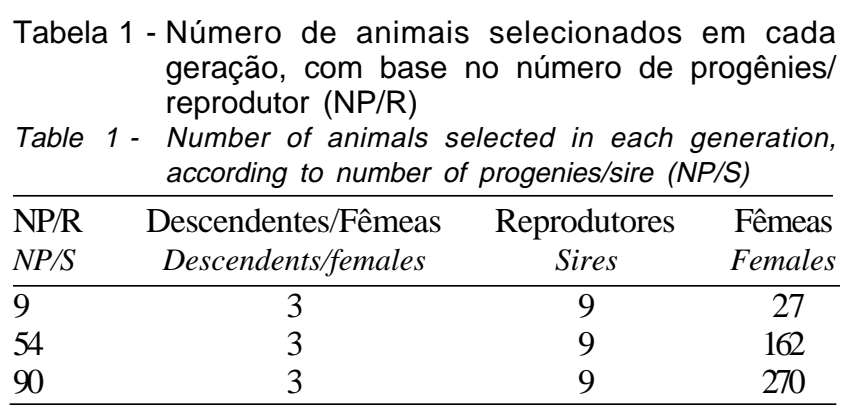




\section{Rev. bras. zootec.}

distribuídas em 2, 3, 6 ou 8 rebanhos. Segundo a metodologia de MATHUR et al. (1999), o grau médio de conexidade para cada arranjo foi calculado como a proporção média de progênies dentro de cada rebanho que são descendentes de reprodutores comuns.

Nas Tabelas 2 e 3 são apresentados exemplos dos tipos de arranjos simulados com diferentes graus de conexidade: um arranjo com $0 \%$ de conexidade, reprodutores com progênies em um único rebanho, e outro com $30 \%$ de conexidade, em que os reprodutores tinham progênies em três rebanhos.

A seleção dos animais foi feita com base nas informações do próprio indivíduo, dos seus ascendentes (pai e mãe) e colaterais (irmãos completos e meioirmãos), uma vez que a matriz de parentesco continha apenas as informações de pedigree relativas às duas últimas gerações. Utilizou-se o modelo animal, descrito a seguir:

$$
\mathrm{y}_{\mathrm{ikk}}=\mu+\mathrm{r}_{\mathrm{i}}+\mathrm{s}_{\mathrm{j}}+\mathrm{a}_{\mathrm{ijk}}+\mathrm{e}_{\mathrm{ijk}}
$$

em que $y_{i k k}$ é observação do animal $i$, do rebanho $\mathrm{j} e$ do sexo $\mathrm{k} ; \mu$, constante associada a cada observação; $r_{i}$, efeito fixo de rebanho, com $i=1,2,3, \ldots, 9$; $\mathrm{s}_{\mathrm{j}}$, efeito fixo de sexo, com $\mathrm{j}=1$ e $2 ; \mathrm{a}_{\mathrm{ijk}}$, efeito aleatório do animal k, no rebanho i e do sexo j; $\mathrm{e}_{\mathrm{ijk}}$, erro aleatório associado a cada observação.

A acurácia das avaliações genéticas foi calculada como a correlação entre os valores genéticos verdadeiros e preditos $\left(\mathrm{r}_{\mathrm{a}, \hat{a}}\right)$, de acordo com a equação descrita a seguir:

$$
\mathrm{r}_{\mathrm{a}, \hat{\mathrm{a}}}=\frac{\operatorname{Cov}(\mathrm{a}, \hat{\mathrm{a}})}{\sqrt{\mathrm{V}(\mathrm{a}) \cdot \mathrm{V}(\hat{\mathrm{a}})}}
$$

em que a é valor genético verdadeiro e â, valor genético predito.

Calculou-se a acurácia do teste de progênie, relativa aos valores genéticos dos nove reprodutores já selecionados, a cada geração, que possuíam progênies. Também foi calculada a acurácia do teste de performance, relativa aos valores genéticos dos animais candidatos à seleção e que ainda não tinham progênies.

\section{Resultados e Discussão}

As médias de acurácia do teste de progênie foram menores que as encontradas por OJALA et al. (1984) e TOSH e WILTON (1994), provavelmente, porque, neste estudo, não foi considerado o parentesco entre reprodutores, uma vez que a matriz de parentesco continha informações de pedigree apenas das duas últimas gerações.

A acurácia do teste de progênie foi fortemente influenciada pela herdabilidade da característica e pelo número de progênies, concordando com o que foi relatado por PEREIRA (1996) sobre os fatores que afetam a eficiência do teste de progênie. Com o aumento da herdabilidade e do número de progênies, os valores de acurácia aumentaram consideravelmente para todos os arranjos simulados. No entanto, o aumento de 54 para 90 progênies resultou em pequenas alterações na acurácia, dentro da mesma herdabilidade (Tabela 4).

A utilização do arranjo de dados com $0 \%$ de conexidade levou aos menores valores de acurácia para todas as situações simuladas (Tabela 4).

Para arranjos com menores números de progênies e baixas herdabilidades, observou-se maior efeito da conexidade sobre a acurácia do teste de progênie. Nestes casos, a superioridade da acurácia dos arranjos conectados, em relação ao arranjo com $0 \%$ de conexidade, foi maior que a observada para arranjos

Tabela 2 - Arranjo com $0 \%$ de conexidade e 90 progênies/reprodutor

\begin{tabular}{|c|c|c|c|c|c|c|c|c|c|}
\hline \multirow{3}{*}{$\begin{array}{l}\text { Reprodutores } \\
\text { Sires }\end{array}$} & \multicolumn{8}{|c|}{$\begin{array}{c}\text { Rebanhos } \\
\text { Herds }\end{array}$} & \multirow{3}{*}{9} \\
\hline & 1 & 2 & 3 & 4 & 5 & 6 & 7 & 8 & \\
\hline & & & & & & & & & \\
\hline 1 & 90 & - & - & - & - & - & - & - & - \\
\hline 2 & - & 90 & - & - & - & - & - & - & - \\
\hline 3 & - & - & 90 & - & - & - & - & - & - \\
\hline 4 & - & - & - & 90 & - & - & - & - & - \\
\hline 5 & - & - & - & - & 90 & - & - & - & - \\
\hline 6 & - & - & - & - & - & 90 & - & - & - \\
\hline 7 & - & - & - & - & - & - & 90 & - & - \\
\hline 8 & - & - & - & - & - & - & - & 90 & - \\
\hline 9 & - & - & - & - & - & - & - & - & 90 \\
\hline
\end{tabular}

Table 2 - Arrange with $0 \%$ of connectedness and 90 progenies/sire 
Tabela 3 - Arranjo com $30 \%$ de conexidade e 90 progênies/reprodutor

Table 3 - Arrange with $30 \%$ of connectedness and 90 progenies/sire

\begin{tabular}{|c|c|c|c|c|c|c|c|c|c|}
\hline \multirow{3}{*}{$\begin{array}{l}\text { Reprodutores } \\
\text { Sires }\end{array}$} & \multicolumn{8}{|c|}{$\begin{array}{c}\text { Rebanhos } \\
\text { Herds }\end{array}$} & \multirow{3}{*}{9} \\
\hline & 1 & 2 & 3 & 4 & 5 & 6 & 7 & 8 & \\
\hline & & & & & & & & & \\
\hline 1 & 30 & 30 & 30 & - & - & - & - & - & - \\
\hline 2 & - & 30 & 30 & 30 & - & - & - & - & - \\
\hline 3 & - & - & 30 & 30 & 30 & - & - & - & - \\
\hline 4 & - & - & - & 30 & 30 & 30 & - & - & - \\
\hline 5 & - & - & - & - & 30 & 30 & 30 & - & - \\
\hline 6 & - & - & - & - & - & 30 & 30 & 30 & - \\
\hline 7 & - & - & - & - & - & - & 30 & 30 & 30 \\
\hline 8 & 30 & - & - & - & - & - & - & 30 & 30 \\
\hline 9 & 30 & 30 & - & - & - & - & - & - & 30 \\
\hline
\end{tabular}

com progênies mais numerosas e altas herdabilidades (Tabela 4). Estes resultados foram semelhantes aos observados por TOSH e WILTON (1994), que encontraram maior importância da conexidade dos dados, quando o número de progênies/reprodutor foi baixo, menor que 10.

A partir de 54 progênies, mesmo para arranjos com $0 \%$ de conexidade, a acurácia do teste de progênie foi superior à obtida com dados $100 \%$ conectados e 9 progênies (Tabela 4), o que revela que o número de progênies tiveram grande efeito sobre a acurácia das avaliações de reprodutores. Resultados similares foram encontrados por OJALA et al. (1984) e TOSH e WILTON (1994)

Na maioria dos casos, o uso de arranjos com mais de $60 \%$ de conexidade levou a alterações pequenas na acurácia do teste de progênie. Para arranjos com 90 progênies e alta herdabilidade $(0,60)$, a partir de $30 \%$ de conexidade, não se observaram quaisquer alterações nos valores de acurácia com o aumento da conexidade.

A acurácia do teste de performance foi inferior à acurácia do teste de progênie, e esta diferença foi maior para progênies mais numerosas (Tabelas 4 e 5), uma vez que a predição dos valores genéticos dos reprodutores se torna mais acurada com o aumento no número de progênies.

Com o aumento da herdabilidade da característica, foi observada redução na diferença entre os valores de acurácia do teste de performance, obtidos para os arranjos simulados com diferentes graus de conexidade (Tabela 5).

As médias de acurácia do teste de performance aumentaram com o aumento do grau de conexidade dos dados e, para os arranjos com 9 progênies, a partir de $60 \%$ de conexidade, pequenas alterações nos valores de acurácia foram observadas (Tabela 5).

Para arranjos com 54 e 90 progênies e herdabilidades 0,10 e 0,30 , a partir de $15 \%$ de conexidade, ocorreram pequenos acréscimos na acurácia do teste de performance, com o aumento no grau de conexidade. Já para características com herdabilidade 0,60 não foi observado efeito do grau de conexidade (Tabela 5).

De modo geral, o grau de conexidade dos dados teve maior influência sobre a acurácia do teste de progênie, em comparação à acurácia do teste de performance. TOSH e WILTON (1994) verificaram pequena correlação entre o grau de conexidade dos dados e a PEV das avaliações de animais sem progênies, enquanto o efeito da conexidade sobre a acurácia do teste de progênie foi alto.

O maior efeito da conexidade dos dados sobre a acurácia do teste de progênie e sobre características de baixa herdabilidade, provavelmente, ocorreu porque nestas duas situações o BLUP atribui maior importância relativa às informações de parentes. Neste caso, a distribuição dos parentes dos indivíduos em maior número de rebanhos, aumentando a conexidade dos dados, terá maior influência nos resultados das avaliações genéticas.

$\mathrm{O}$ pequeno efeito da conexidade para arranjos com grande número de progênies parece estar relacionado com o número de animais dentro de cada rebanho. Neste trabalho, o aumento no número de progênies/reprodutor foi acompanhado de acréscimo no tamanho dos rebanhos. Segundo KENNEDY e TRUS (1993), o aumento na PEV das comparações 
346 Rev. bras. zootec.

Tabela 4 - Acurácia média do teste de progênie nas dez gerações, considerando arranjos de dados com 0, 15, 30, 60, 90 e $100 \%$ de conexidade

Table 4 - Average accuracy of the progeny test on the ten generations, for data arranges with 0, 15, 30, 60, 90 and 100\% of connectedness Graus de conexidade

Degrees of connectedness

\begin{tabular}{|c|c|c|c|c|c|c|}
\hline & $0 \%$ & $15 \%$ & $30 \%$ & $60 \%$ & $90 \%$ & $100 \%$ \\
\hline Herdabilidade & \multicolumn{6}{|c|}{9 progênies } \\
\hline Heritability & \multicolumn{6}{|c|}{9 progenies } \\
\hline 0,10 & 14,30 & 17,49 & 23,12 & 27,78 & 27,01 & 28,31 \\
\hline 0,30 & 31,18 & 33,72 & 40,82 & 45,83 & 47,71 & 46,02 \\
\hline 0,60 & 39,46 & 46,57 & 49,22 & 55,97 & 53,98 & 58,09 \\
\hline Herdabilidade & \multicolumn{6}{|c|}{54 progênies } \\
\hline Heritability & \multicolumn{6}{|c|}{54 progenies } \\
\hline 0,10 & 47,21 & 57,77 & 58,32 & 66,32 & 65,41 & 67,07 \\
\hline 0,30 & 57,53 & 71,34 & 75,28 & 78,92 & 79,73 & 79,91 \\
\hline 0,60 & 65,62 & 76,04 & 78,63 & 80,93 & 83,28 & 83,69 \\
\hline Herdabilidade & \multirow{2}{*}{\multicolumn{6}{|c|}{$\begin{array}{l}90 \text { progênies } \\
90 \text { progenies }\end{array}$}} \\
\hline Heritability & & & & & & \\
\hline 0,10 & 46,09 & 63,49 & 65,94 & 67,34 & 67,46 & 68,51 \\
\hline 0,30 & 61,17 & 78,01 & 77,51 & 80,88 & 80,4 & 82,29 \\
\hline 0,60 & 73,36 & 81,45 & 84,85 & 84,37 & 84,71 & 84,57 \\
\hline
\end{tabular}

Tabela 5 - Acurácia média do teste de performance nas dez gerações, considerando arranjos de dados com 0, 15, 30, 60, 90 e $100 \%$ de conexidade

Table 5 - Average accuracy of the performance test in the ten generations, for data arranges with $0,15,30,60,90$ and $100 \%$ of connectedness

\begin{tabular}{|c|c|c|c|c|c|c|}
\hline & \multicolumn{6}{|c|}{$\begin{array}{c}\text { Graus de conexidade } \\
\text { Degrees of connectedness }\end{array}$} \\
\hline & $0 \%$ & $15 \%$ & $30 \%$ & $60 \%$ & $90 \%$ & $100 \%$ \\
\hline Herdabilidade & \multicolumn{6}{|c|}{9 progênies } \\
\hline Heritability & \multicolumn{6}{|c|}{9 progenies } \\
\hline 0,10 & 15,79 & 17,47 & 18,27 & 21,19 & 22,36 & 21,71 \\
\hline 0,30 & 21,94 & 21,78 & 23,34 & 26,5 & 27,8 & 28,15 \\
\hline 0,60 & 30,87 & 31,65 & 33,13 & 35,27 & 37,4 & 36,87 \\
\hline Herdabilidade & \multirow{2}{*}{\multicolumn{6}{|c|}{$\begin{array}{l}54 \text { progênies } \\
54 \text { progenies }\end{array}$}} \\
\hline Heritability & & & & & & \\
\hline 0,10 & 16,82 & 21,26 & 19,80 & 22,36 & 22,36 & 23,05 \\
\hline 0,30 & 31,08 & 32,67 & 32,66 & 33,71 & 33,34 & 32,74 \\
\hline 0,60 & 46,07 & 45,12 & 44,10 & 44,99 & 45,55 & 44,77 \\
\hline Herdabilidade & \multirow{2}{*}{\multicolumn{6}{|c|}{$\begin{array}{l}90 \text { progênies } \\
90 \text { progenies }\end{array}$}} \\
\hline Heritability & & & & & & \\
\hline 0,10 & 7,93 & 15,35 & 15,27 & 15,62 & 16,34 & 17,05 \\
\hline 0,30 & 27,39 & 31,60 & 32,25 & 31,91 & 32,68 & 33,15 \\
\hline 0,60 & 49,97 & 50,01 & 50,25 & 50,29 & 50,72 & 51,32 \\
\hline
\end{tabular}

entre valores genéticos de animais pertencentes a diferentes rebanhos é pequeno, quando se trabalha com rebanhos com grande número de animais.

Os resultados deste estudo mostraram que o grau de conexidade dos dados foi mais importante para características de baixa herdabilidade. A baixa conexidade dos dados, provavelmente, aumentou a variância da diferença entre as estimativas dos efeitos fixos de rebanho. Assim, como as características de baixa herdabilidade são afetadas com maior intensidade pelas variações ambientais, a avaliação destas caraterísticas tenderam a ser mais influenciadas pela conexidade dos dados. 


\section{Conclusões}

O grau de conexidade dos dados teve maior influência sobre a acurácia do teste de progênie que sobre a acurácia do teste de performance.

A baixa conexidade dos dados, principalmente o uso de dados totalmente desconectados, reduziu a acurácia das avaliações genéticas. De acordo com os resultados encontrados neste trabalho e considerando que, em média, são avaliadas 20 progênies por reprodutor, pode-se inferir que o valor mínimo de conexidade para a execução de uma avaliação genética seja de $15 \%$, para características de média e alta herdabilidade, e de $30 \%$, para características de baixa herdabilidade.

A herdabilidade da característica avaliada e o número de progênies tiveram maior influência na acurácia da avaliação genética que a conexidade dos dados.

A importância da conexidade foi maior nas avaliações genéticas de animais com número reduzido de informações de parentes descendentes ou colaterais e para características de baixa herdabilidade.

\section{Referências Bibliográficas}

BANOS, G. 1994. International genetic evaluation of dairy cattle. Uppsala, Sweden: INTERBULL Centre, Swedish University of Agricultural Sciences. p.1-10.

EUCLYDES, R.F. Uso do sistema para simulação Genesys na avaliação de métodos de seleção clássicos e associados a marcadores moleculares. Viçosa, MG: UFV, 1996. 149p. Dissertação (Doutorado em Genética e Melhoramento) Universidade Federal de Viçosa, 1996.

FOULlEY, J.L., BOUIX, J., GOFFINET, B. et al. 1987. Connectedness in genetic evalution. In: GIANOLA, D., HAMMOND, K., (Eds.) Advances in statistical methods for genetic improvement of livestock. Armidale, Austrália: ABGU, p.302-337.

FRIES, L.A., ROSO, V.M. Conectabilidade em avaliações genéticas de gado de corte: uma proposta heurística. In: REUNIÃO ANUAL DA SOCIEDADE BRASILEIRA DE ZOOTECNIA, 34, 1997, Juiz de Fora. Anais... Juiz de Fora: EMBRAPA/CNPGL, 1997. v.3, p.159-161.

KENNEDY, B.W., TRUS, D. 1993. Considerations on genetic connectedness between management units under an animal model. J. Anim. Sci., 71(9):2341-2352.

MARTINS, E.N. Uso de modelos mistos no melhoramento animal. In: SIMPÓSIO INTERNACIONAL DE PRODUÇÃO DE NÃO-RUMINANTES, 1994, Maringá. Anais... Maringá: SBZ, 1994. p.41-46.

MATHUR, P.K., SULLIVAN, B., CHESNAIS, J. "Estimation of the degree connectedness between herds or management groups in the canadian swine population", <http:// www.ccsi.ca/pramod/connectedness.htm> (07/01/99).

OJALA, M., SYVAJARVI, J., HELLMAN, T. 1996. Effect of the size of the comparison on the accuracy of a sire's evaluation. J. Anim. Breeding, 102:91-94.

PEREIRA, J.C.C. 1999. Melhoramento genético aplicado à produção animal. Belo Horizonte. 416p.

PHILIPSSON, J. 1987. Standards and procedures for international genetic evaluations of dairy cattle. J. Dairy. Sci., 70(2):418-424.

SCHENKEL, L.R. Aplicação da metodologia dos modelos mistos na avaliação genética de suínos. Porto Alegre, RS: UFRGS, 1991. 207p. Dissertação (Mestrado em Zootecnia) Universidade Federal do Rio Grande do Sul/Faculdade de Agronomia, 1991.

TOSH, J.J., WILTON, J.W. 1994. Effects of data struture on variance of prediction error and accuracy of genetic evaluation. J. Anim. Sci., 72:2568-2577.

Recebido em: 10/02/00

Aceito em: 23/11/00 